einfach in die Vorlagen überdestillirt sei. In diesem Falle musste also der Zinkstaub cyankaliumbaltig sein. Andererseits konnte sich aber auch eine bei dieser Temperatur flüchtige Cyanzinkverbindung schon in der Muffel gebildet haben, die ebenfalls in den kühleren Vorlagen sich wieder verdichtete. Oder aber die Cyanbildung vollzog sich überhaupt erst in den kühleren Theilen, namentlich in den Zinkstaubrorlagen, in die ja schon ein reichlicher Zutritt von atmosphärischer Luft erfolgt. In diesem Falle würde die Entstehung der Cyanide zurückzuführen sein auf die unmittelbare Einwirkung von Stickstoff auf das Gemisch von Dämpfen metallischen Zinks mit Kohlenoxydulgas und nach folgender Gleichung stattfinden:

$3 \mathrm{Zn}+2 \mathrm{CO}+\mathrm{N}_{2}=\mathrm{Zn}(\mathrm{CN})_{2}+2 \mathrm{ZnO}$.

Áusserdem lag aber auch noch die Möglichkeit vor, dass das fein vertheilte Zinkoxyd bei Gegenwart der Metalldämpfe auf überdestillirtes Kaliumcyanid eingewirkt und nachstehende Umsetzung herbeigeführt hätte: $\mathrm{ZnO}+2 \mathrm{~K}(\mathrm{CN})_{2}=\mathrm{Zn}(\mathrm{CN})_{2}+\mathrm{K}_{2} \mathrm{O}$.

Angesichts der grossen Mengen staubförmigen Zink und Zinkoxydes gegenüber den Spuren von Cyan konnte dieser Nachweis bez. die Trennung etwaiger Alkalicyanide von den entsprechenden Metallverbindungen nicht mittels Wassers bewirkt werden. Da aber Ätzkali sich auch in wasserfreiem Alkohol leicht löst, die Cyanide, $\mathrm{Zn}$ und $\mathrm{Zn} \mathrm{O}$ dagegen darin unlöslich sind, so sollte durch Auslaugen dieses Zinkstaubes mittels des letzgenannten Lösungsmittels dargethan werden, ob eine Umsetzung von Kaliumcyanid mit Zinkoxyd eingetreten wäre. In Folge dessen wurden $300 \mathrm{~g}$ Zinkstaub der Auslaugung unterworfen, Kaliumoxyd war jedoch in dem abgezogenen Alkohol nicht nachweisbar, auch konnte derselbe ohne Rückstand überdestillirt werden.

Eine Umsetzung nach der letzten Gleichung hatte demgemäss nicht stattgefunden. Da ferner der ausgelaugte Rückstand, wie eine Flammenreaction ergab, alkalifrei war, so konnte auch nicht Kaliumcyanid unmittelbar aus der Muffel in die Vorlage verdampft sein - die vorhandene Cyanverbindung musste also zinkhaltig sein.

Bei längerem Kochen dieses Zinkstaubes mit cyanfreier Natronlauge konnte in dem Filtrat mittels Silbernitrats deutlich Cyan nachgewiesen werden. Da das Material frei von Erdalkalien war und man das Blei in Form des Sulfats erhielt, so ist also das Vorhandensein eines Zinkcyanids anzunehmen, doch bleibt die Entstehung desselben noch unaufgeklärt.
Wirkung der Schwefelalkalien auf die Lösungen der Metalle der Eisengruppe.

\author{
Vorläufige Mittheilung \\ von \\ Prof. Dr. L. L. de Koninck \\ und \\ M. Ledent, \\ Candidat Scient.
}

In den Lehrbüchern der analytischen Chemie, von H. Rose's Handbuchs ersten Ausgaben an bis zu den allerneuesten, wird angegeben, dass das Schwefelammon in den Lösungen von Eisen, Mangan, Zink, Nickel und Kobalt einen Niederschlag von Schwefelmetallen hervorbringt; die einzigen angeführten Eigenthümlichkeiten dieser Reactionen lauten folgendermaassen:

1. Es bleibt bisweilen etwas Schwefeleisen in Suspension; in diesem Falle ist die Flüssigkeit grün, aber nach längerem Stehen verschwindet diese Färbung, und die Fällung des Eisensulfürs wird vollständig.

2. Die Fällung des Mangans ist nicht vollständig; Spuren dieses Metalles findet man im rom Niederschlage getrennten Filtrate, selbst dann, wenn das Filtrat durchaus klar ist.

3. Das Schwefelnickel ist oft in einem Übermaasse von Schwefelammonium etwas löslich; die Auflösung ist braun. Bei den Kobaltsalzen kommt diese Erscheinung nicht vor.

In Betreff der Wirkung des Schwefelkaliums und Schwefelnatriums wird gewöhnlich nichts erwähnt, oder, wenn davon die Rede ist, vermischt man diese Reagentien mit dem - Schwefelammon unter der Gemeinbezeichnung von Schwefelalkalien.

Wir kennen keine Abhandlung über die Wirkung der fixen Schwefelalkalien auf die ebengenannten Metallsalze.

Die einzigen, unseres Wissens diesbezüglichen Arbeiten über die Wirkung des Schwefelammons sind folgende: erstens eine schon alte und sehr oberfächliche Notiz von Terreil ${ }^{1}$ ), nach welcher, wenn Ammoniaksalze und Ammoniak im Überschuss vorhanden sind, die Zink-, Mangan-, Nickel- und Kobaltsalze nicht oder doch sebr unvollständig von Schwefelammon gefällt werden; die Nickel- bez. Kobaltlösungen wären beide mahagonibraun $^{2}$ ). Nach Terreil wäre im Gegentheil die Fällung der Ferrosalze (der

1) Compt. r. 45 S. 652 (1857).

2) War das angewandte Kobaltsalz vollständig nickelfrei? 
Verfasser erwähnt nicht die Ferrisalze) unter diesen Bedingungen eine vollständige.

C. Meineke (d. Z. 1888, 4) hat untersucht, was wir oben erwähnen in Betreft des Manganniederschlags und festgestellt, dass selbst dann, wenn das Mangansulfür wasserfrei und grün geworden ist, einige Zehntel Milligr. noch in Lösung bleiben.

Wir erwähnen schliesslich eine neuere Scbrift von Dr. Ad, Lecrenier (Chemztg. 1889 , No. 27 u. 28) über die Auflöslichkeit des Schwefelnickels im Schwefelammonium. Gestützt auf diese Arbeit, wonach die Auflöslichkeit des Schwefelnickels dem Schwefelüberschusse des Reagens zu verdanken wäre, erschien es uns interessant zu untersuchen, was wir für wahrscheinlich hielten, ob sich dieselbe Erscheinung nicht mit den Natrium- bez. Kaliumpolysulfiden erzielen liesse, welch letztere weniger veränderlich als Schwefelammonium sind, und daun ferner auch zu sehen, ob die anderen Metalle dieser Gruppe und namentlich Kobalt, dem Nickel so verwandt, das Eisen und sogar Zink und Mangan, sich nicht gleich dem Nickel verhalten würden.

Aus etlichen Versuchen, die wir unternommen haben, geht hervor, dass unsere Voraussetzungen wenigstens zum Theil zutreffend waren; die Nickel-, Kobalt- und Eisensalze, mit einem Überschusse von $\mathrm{Na-}$ triumpolysulfid behandelt, erzeugen alle drei äusserst dunkle aber dennoch klare Flüssigkeiten, welche vollständig durch das beste Filtrirpapier gehen, also, scheinbar wenigstens, aufgelöstes Schwefelmetall entbalten, wie es beim Nickel mit Schwefelammonium der Fall ist.

Mit Nickel erscheint die Auflösung schwärzlich-braun, gerade so wie mit Schwefelammonium. Mit den Kobaltsalzen ist die Färbung etwas verschieden, schmutzig violettbraun.

Mit Eisen endlich geht die Aufösung von einer dunkelbraunen unbeschreiblichen Färbung allmählich in lebbaftes Grün über, an die Färbungen der Auflösungen des Chromsulfats oder des Kaliummanganats erinnernd. Diese Färbung wurde schon früher beobachtet und der eine von $u^{*} s^{3}$ ) deutete schon auf diese äusserst empfindliche Reaction für Eisensalze hin.

Die Zink- und Mangansalze erzeugen keine besondere Färbung; die Schwefelverbindungen dieser beiden Metalle erscheinen unlöslich in Natriumpolysulfid; dieselben sind doch mindestens weit weniger löslich als

3) L. L. de Koninck: Exercices d'analyse chimique qualitative 1885 S. 47. diejenigen der Metalle, welche schwarzen Sulfiden entsprechen.

Da wir genöthigt sind, die nähere Untersuchung dieser Erscheinungen auf eivige Monate rerschieben zu müssen, veröffentlichen wir vorläufig diese Resultate, in der Hoffnung, dass man uns weitere Arbeiten in dieser Richtung überlassen wird.

6. Mittheilung aus dem agriculturchemischen Laboratorium der Universität München.

\section{Neue Methoden der quantitativen Analyse.}

$$
\text { Von }
$$

\section{Anton Baumann.}

In der letzten Mittheilung habe ich das Verhalten der Chromsäure zu Wasserstoffsuperoxyd kurz besprochen und einige exacte und äusserst einfache Methoden der quantitativen Analyse angegeben, welche auf die Reactionsfähigkeit beider Substanzen sich gründen. Eine grosse Anzahl von Versuchen sind inzwischen im hiesigen Laboratorium angestellt worden, um die gleiche Reaction zur quantitativen Analyse verschiedener $\mathrm{Me}$ talle und gewisser organischer Körper zu verwerthen.

Indem ich den Bericht über diese Arbeiten der nächsten Mittheilung vorbehalte, möchte ich zur Aufstellung neuer analytischer Methoden auf das Verhalten des Wasserstoffsuperoxyds gegen Jod hinweisen, welches in gleicher Weise wie die Reaction gegen Chromsäure für die quantitative Analyse von grosser Bedeutung zu werden verspricht.

In der Litteratur sind über diesen Gegenstand nur sehr spärliche Angaben aufzufinden. Ich war deshalb hinsichtlich der Begründung der in der Folge beschriebenen Methoden grösstentheils auf eigene Versuche angewiesen und ich werde die Resultate derselben hier so weit mittheilen, als es zum Verständniss und zur richtigen Ausfübrung der Analysen nothwendig erscheint.

Bekannt ist bereits, dass beim Zusammentreffen von Wasserstoffsuperoxyd mit Jod in wässeriger und saurer Lösung sich Sauerstoff entwickelt. Man pflegt anzunehmen, dass diese Reaction nach der Gleichung verlaufe: $\mathrm{J}_{2}+\mathrm{H}_{2} \mathrm{O}_{2}=\mathrm{O}_{2}+\mathrm{JH}$, und man könnte demgemäss erwarten, dass beim Behandeln einer wässerigen Jodlösung mit Wasserstoff- 